\title{
Retinal redox stress and remodeling in cardiometabolic syndrome and diabetes
}

\author{
Ying Yang, ${ }^{1,3-5}$ Melvin R. Hayden, ${ }^{2,4, *}$ Susan Sowers, ${ }^{4,5}$ Sarika V. Bagree ${ }^{2,4}$ and James R. Sowers ${ }^{2-5}$ \\ ${ }^{1}$ Yunnan Province $2^{\text {nd }}$ Hospital; Kunming, PR China; ${ }^{2}$ University of Missouri School of Medicine; Department of Internal Medicine; ${ }^{3}$ Physiology and Pharmacology; ${ }^{4}$ Division of \\ Endocrinology, Diabetes, Metabolism and Cardiovascular Disease; ${ }^{5}$ Harry S. Truman VA Medical Center; MO USA
}

Key words: retina, redox stress, cardiometabolic syndrome, type 2 diabetes mellitus

Diabetic retinopathy (DR) is a significant cause of global blindness; a major cause of blindness in the United States in people aged between $20-74$. There is emerging evidence that retinopathy is initiated and propagated by multiple metabolic toxicities associated with excess production of reactive oxygen species (ROS). The four traditional metabolic pathways involved in the development of DR include: increased polyol pathway flux, advanced glycation end-product formation, activation of protein kinase Cisoforms and hexosamine pathway flux. These pathways individually and synergisticallycontribute to redox stress with excess ROS resulting in retinal tissue injury resulting in significant microvascular blood retinal barrier remodeling. The toxicity of hyperinsulinemia, hyperglycemia, hypertension, dyslipidemia, increased cytokines and growth factors, in conjunction with redox stress, contribute to the development and progression of DR. Redox stress contributes to the development and progression of abnormalities of endothelial cells and pericytes in DR. This review focuses on the ultrastructural observations of the blood retinal barrier including the relationship between the endothelial cell and pericyte remodeling in young nine week old Zucker obese ( $\mathrm{fa} /$ fa) rat model of obesity; cardiometabolic syndrome, and the 20 week old alloxan induced diabetic porcine model. Preventing or delaying the blindness associated with these intersecting abnormal metabolic pathways may be approached through strategies targeted to reduction of tissue inflammation and oxidative-redox stress. Understanding these abnormal metabolic pathways and the accompanying redox stress and remodeling mayprovide both the clinician and researcher a new concept of approaching this complicated disease process.

\section{Introduction}

Diabetic retinopathy (DR) is a microvascular complication that presages development of type 2 diabetes mellitus (T2DM) and accounts for blindness in over 10,000 people each year. ${ }^{1}$ Data from the National Eye Institute has demonstrated that half of the patients with diabetes in the United States have some form of retinopathy, and approximately 700,000 have some type of serious retinal disease. ${ }^{1}$ There is a growing incidence of T2DM, which accounts for about $90 \%$ of the 24 million cases of diabetes

*Correspondence to: Melvin R. Hayden; Email: mrh29@usmo.com Submitted: 12/13/10; Revised: 01/04/11; Accepted: 01/07/11

DOI: 10.4161/oxim.3.6.14786 mellitus in the United States. A cluster of metabolic abnormalities related to the cardiometabolic syndrome (CMS) including central obesity, metabolic dyslipidemia, insulin resistance (IR) and hypertension increase the risk for T2DM and DR. Risk factors for DR are often present in insulin resistant, hypertensive persons prior to the development of T2DM. ${ }^{1-5}$ Several epidemiological studies have yielded various metabolic and systemic inflammatory factors associated with the epidemiology of DR. ${ }^{6,7}$ These factors are associated with inflammation and increased tissue generation of reactive oxygen species (ROS) that help drive the retinal remodeling process. ${ }^{2,3}$ Excessive accumulation of retinal ROS helps drive cellular and extracellular matrix (ECM) remodeling, and a pivotal mechanism in the development of retinal disease in association with the CMS..$^{3-13}$

There are four main molecular mechanisms implicated in glucose-mediated vascular damage. These classic mechanisms include increased polyol pathway flux, ${ }^{9}$ increased advanced glycation end-products (AGE) formation, activation of protein kinase $\mathrm{C}$ (PKC) isoforms and increased hexosamine pathway flux. ${ }^{10}$ Each of these different mechanisms involves the overproduction of superoxide anions: processes involving mitochondrial electron transport chain uncoupling, the formation of AGE and its receptor, RAGE and increased $\mathrm{NAD}(\mathrm{P}) \mathrm{H}$ oxidase activation. ${ }^{11}$ The current overview of the pathophysiology of metabolic/diabetic retinopathy will focus on structural abnormalities accompanying the abnormal retinal metabolic milieu in the CMS.

\section{Diabetic Retinopathy (DR)}

DR is associated with the following structural remodeling features: basement membrane (BM) thickening, pericyte loss, microaneurysms, intraretinal microvascular abnormalities (IRMA), diabetic macular edema (DME) and pre-retinal neovascularization, processes which can lead to blindness through hemorrhage and tractional retinal detachment. ${ }^{13}$ Retinal endothelial cells (EC) are supported and sealed by a nearly equal number of pericytes in the retinal optic nerve fiber, inner and outer plexiform and choroidal layers creating a blood retinal barrier (BRB) of closed capillaries (Figs. 1 and 2). ${ }^{14-16}$

Pericyte degeneration (ghost cells) and/or loss (dropout) (Fig. 3) are considered one of the earliest ultrastructural hallmarks along with capillary basement membrane (CBM) thickening to be identified with DR. In diabetic rodents, the earliest morphological change is reduction of pericyte numbers manifested 


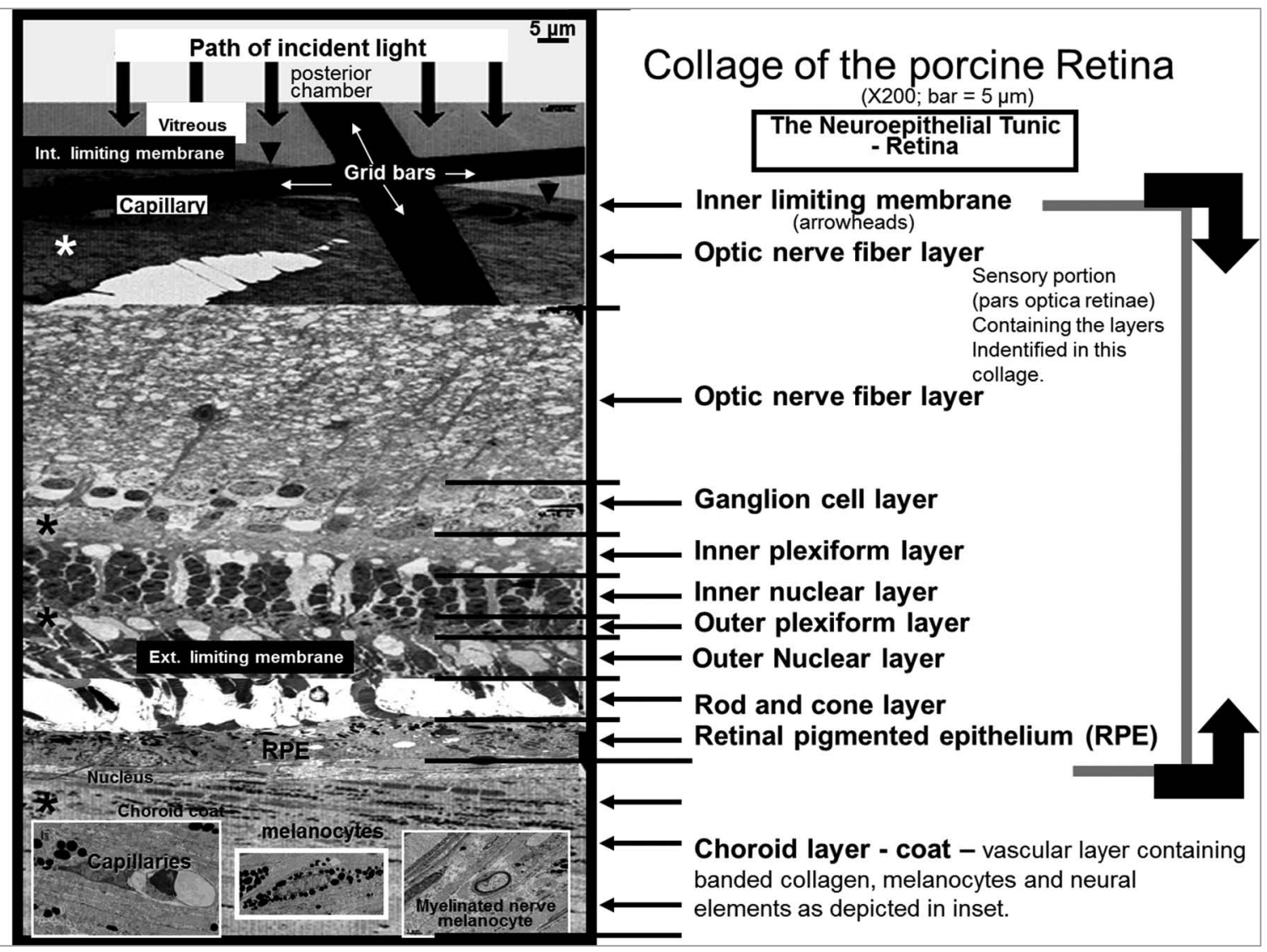

Figure 1. Collage of the Porcine Retina. This collage of the retina allows one to follow the path of incident light as it passes through each layer of the retina and into the choroid coat. Each layer of the retina is prominently displayed and identified layer by layer.

by degeneration (ghost cells) or loss in retinal capillaries, followed by increased numbers of acellular-occluded capillaries, incidental microaneurysms and CBM thickening. Visual loss or sight-threatening complications, results from neovascularization-proliferative diabetic retinopathy (PDR) and/or increased retinal microvascular permeability resulting in increased ECM hydrostatic pressure and DME. ${ }^{12}$ In the human diabetic eye, severe visual loss is often associated with macula edema due to a progressive increase of vascular permeability and/or tractional retinal detachment by the new blood vessels of PDR and contraction of the accompanying fibrous tissue (ECM) or neovascularization, which may hemorrhage, adding to the further complication of pre-retinal or vitreous hemorrhage. Importantly, DME is the main cause of central vision loss, neovascularization, vitreous hemorrhage, and retinal detachment in DR patients (Fig. 4).

As iterated above, the polyol pathway, ${ }^{9}$ non-enzymatic glycation, ${ }^{10}$ increased hexosamine flux, ${ }^{10}$ and activation of PKC are associated with excessive ROS and antioxidant depletion (Fig. 5). The polyol pathway includes the conversion of glucose into sorbitol catalyzed by the aldose reductase enzyme, and sorbitol is then oxidized to fructose by the sorbitol dehydrogenase enzyme.,10 An activated polyol pathway in DR could enhance redox stress because aldose reductase requires $\mathrm{NAD}(\mathrm{P}) \mathrm{H}$ and $\mathrm{NAD}(\mathrm{P}) \mathrm{H}$ is consumed, decreasing its availability for regenerating the intracellular antioxidant, glutathione (GSH).$^{13}$ ROS are generated via the AGE pathway leading to the activation of redox-sensitive transcription factors causing further damage to retinal cells. In DR accumulation of AGE and its receptor, RAGE, is increased in the retinal microvasculature. ${ }^{14}$ In the CMS there is increased hexosamine pathway activity, which enhances fructose- 6 phosphate, which provides substrates for reactions requiring uridine diphosphate-N-(UDP-N-) acetylglucosamine, while promoting DR. ${ }^{10}$

All four key metabolic pathways (polyol, PKC, hexosamine flux and AGE pathways) are activated by an excess glucose resulting in intracellular accumulation of glyceraldehyde-3 phosphate. Excess glyceraldehyde- 3 phosphate is further enhanced due to increased ROS and subsequent accumulation of poly ADP ribose polymerase (PARP), which contributes to activation of the PKC pathway, as well as increasing AGE formation and cellular damage. ${ }^{10,11}$ Increased PKC activity and associated increases in ROS are important in the pathogenesis of metabolic DR. Glycosylated proteins generate free radicals, leading to oxidative tissue damage and depletion of GSH, which is decreased in the retinas of diabetic animals. ${ }^{15}$ Additionally, glycosylated proteins may combine with lipids forming advanced lipoxidation end products, which can deposit in blood vessels of the retina and contribute to neovascularization, a process partially blocked by antioxidants. ${ }^{16}$ Patients with DR have higher levels of markers associated with oxidative damage in the sub-retinal fluid as compared to those 


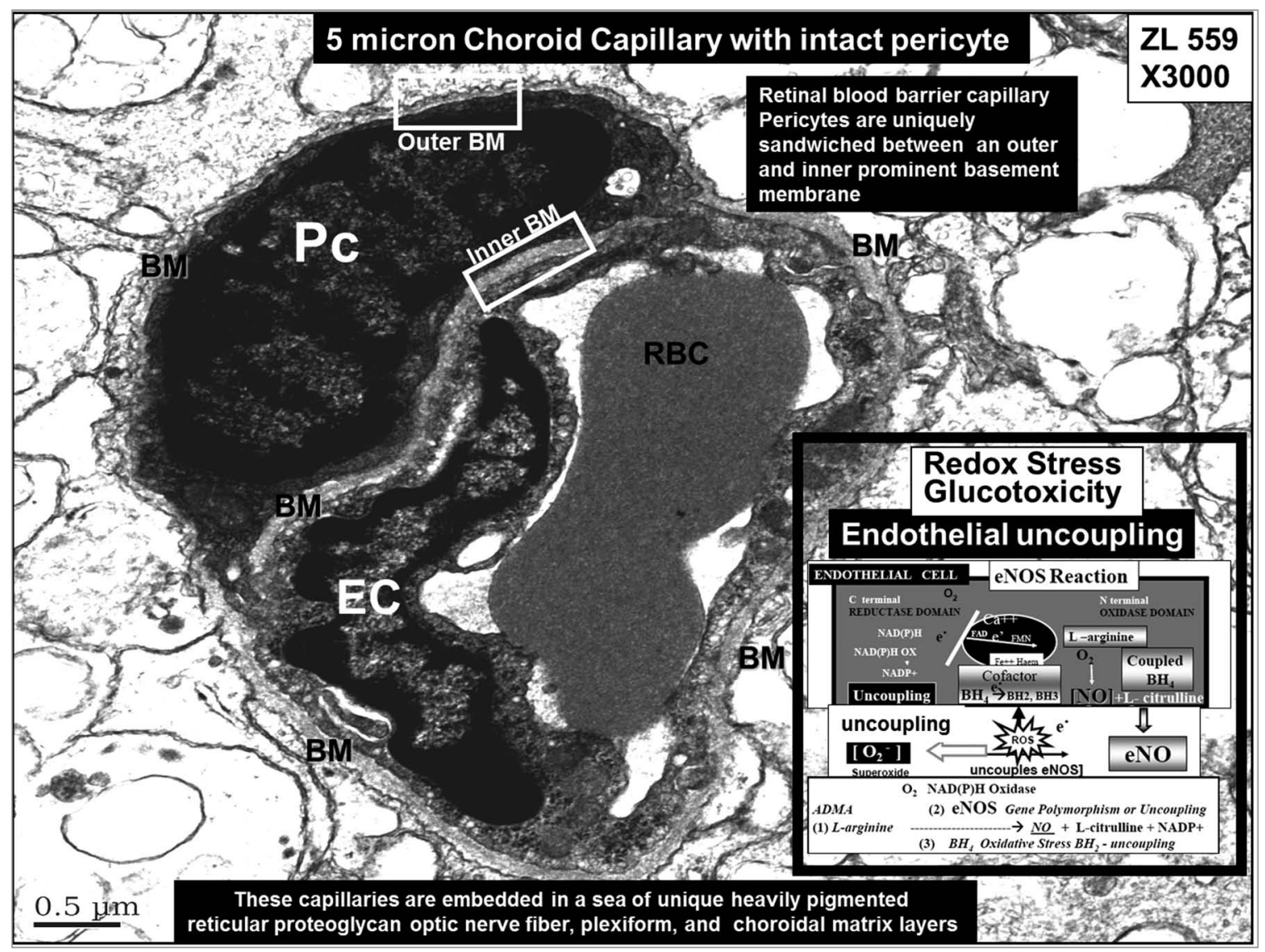

Figure 2. Five Micron Choroid Capillary with Intact Pericyte. This image depicts the normal relationship of the pericyte (PC) in the blood-retinal barrier in relation to the endothelial cell (EC). Note the red blood cell (RBC) within its capillary lumen. The inner and outer basement membranes (BM) are boxed in and identified. Inset depicts how endothelial uncoupling occurs.

without retinopathy. ${ }^{17}$ Additionally, the retina consumes large amounts of polyunsaturated fatty acids and has the highest oxygen uptake and glucose oxidation relative to any other tissue indicating that the retina may be more susceptible to oxidative stress. In summary, alterations in the redox/oxidative stress are key to the pathogenesis of DR.

\section{Redox Stress}

ROS includes the following oxygen free radicals [superoxide $\left(\mathrm{O}_{2}^{-}\right)$, hydrogen peroxide $\left(\mathrm{H}_{2} \mathrm{O}_{2}\right)$, hydroxyl radical $\left(-\mathrm{OH}^{-}\right)$and singlet oxygen]. Redox homeostasis implies the normal physiologic process of reduction and oxidation in order to repair unstable and damaging ROS (whether generated via oxidation or reduction) and organic analogues, which include reactive nitrogen species (RNS), primarily peroxynitrite (ONOO-). This homeostatic balance is lost (redox-oxidative imbalance) when ROS exceeds the antioxidant capacity of the cell.5,6 Excess ROS are capable of modifying both structure and function as a result of injury to lipids loss of membrane function and increased permeability, as well as modifying nucleic acids (damage to DNA leading to apoptosis and mutations) and proteins (leading to stiff aged proteins and specifically collagen by increased cross-linking of sulfhydryl rich proteins) in DR. ${ }^{18}$

\section{Pericyte Loss}

The exact cause of pericyte loss during early DR is unclear; however, redox stress is thought to be involved. Mitochondrial overproduction of ROS and glucose autoxidation, which leads to increased formation of AGE, activation of PKC, aldose reductase and the nuclear transcription factor $\mathrm{NF \kappa} \beta^{9-11}$ are implicated in pericyte loss. The enzyme aldose reductase, which converts glucose to sorbitol, is expressed in retinal pericytes and ECs. AGE accumulate in pericytes of diabetic animals and may predispose pericytes to phagocytosis. Additionally, damage of pericytes in CMS and diabetes may also be promoted by exposure to oxidatively modified low density lipoprotein (LDL).

Pericytes usually die in very early stages of DR and this process may occur in microvessels that otherwise appear histologically normal (Fig. 3). ${ }^{19}$ Pericyte apoptosis is associated with increased levels of PKC activity and redox stress, Pericyte apoptosis occurs in pericytes cultured in either high glucose or galactose suggesting involvement of the polyol pathway. Aldose reductase inhibitors prevent the selective degeneration of retinal capillary pericytes related to increased aldose reductase activity in pericytes cultured in high galactose media. ${ }^{20}$ Moreover, accelerated microvascular cell death in galactosemic rats, combined with evidence that elevated glucose levels induce DNA damage ${ }^{21}$ and apoptosis suggest an initiating role of high levels of hexose in the demise of retinal pericytes and EC. ${ }^{19}$ 

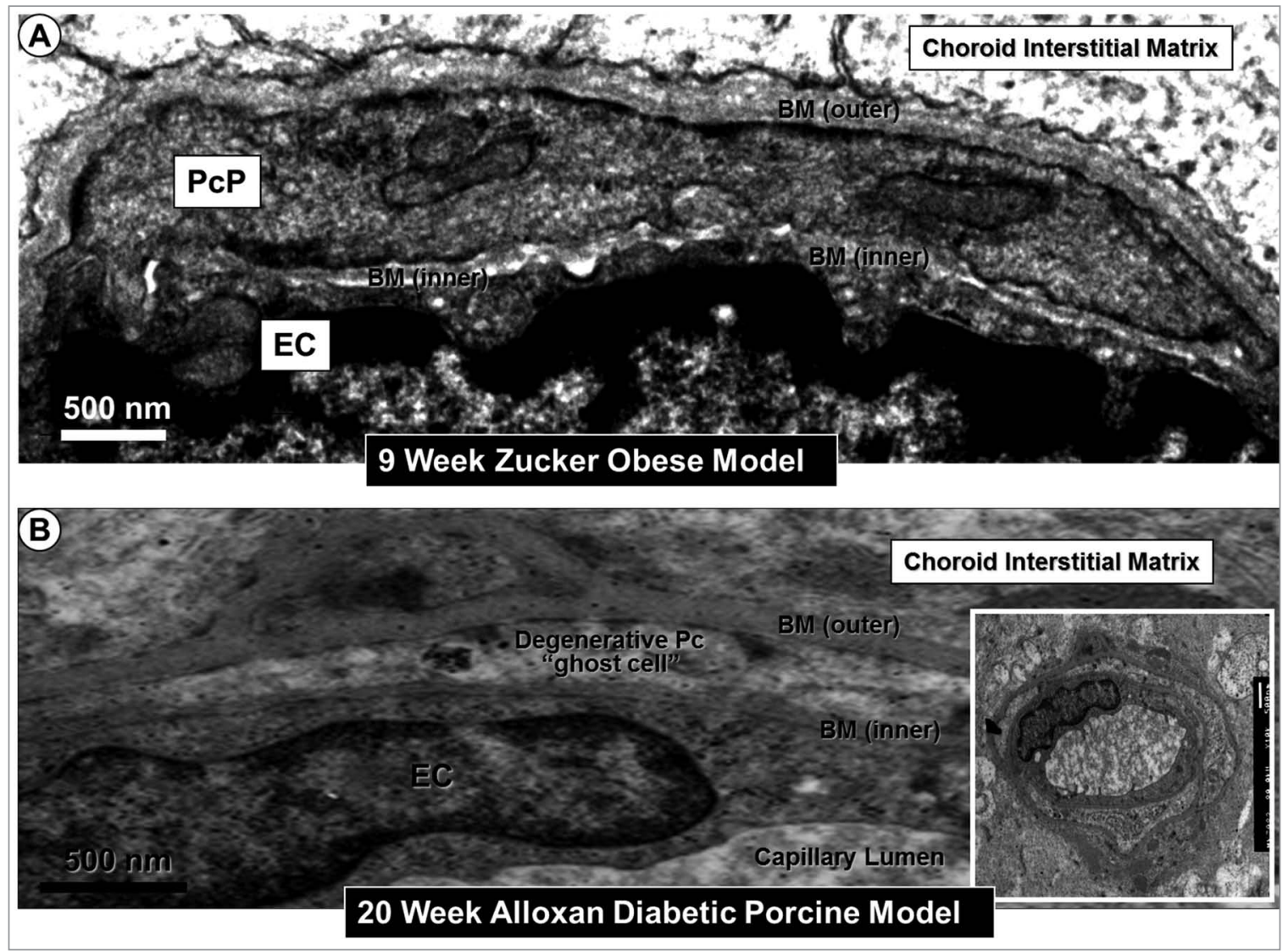

Figure 3. Degenerative Pericyte "Ghost cell" in the 20 Week Alloxan Diabetic Porcine Model. (A) demonstrates the sharply demarcated pericyte process (PCP) sandwiched and encapsulated by an inner and outer basement membrane (BM) as it surrounds the endothelial cell (EC). (B) in contrast depicts PCP degeneration and ghost cell changes typical of the 20 week alloxan diabetic porcine model. Note how the PcP is diminished in size and there is a considerable decrease in electron density staining, typical of pericyte ghost cells. As this progresses the PcP will disappear and its remains are thought to be incorporated within the inner and outer BM to result in BM thickening.

\section{Endothelial Nitric Oxide Synthase (eNOS), Endothelial Derived Nitric Oxide (eNO), and Inducible NOS (iNOS)}

eNOS activation, responsible for the production of eNO, mediates arginine converting it to citrulline and nitric oxide (NO) in EC.5,18 eNOS activation requires the essential cofactor tetrahydrobiopterin (BH4) in its completely reduced form. If $\mathrm{BH} 4$ is oxidized to $\mathrm{BH} 3-\mathrm{BH} 2$ the eNOS enzyme will uncouple and the endothelium will generate excessive ROS (superoxide- $\mathrm{O}_{2}^{-}$) capable of quenching $\mathrm{NO}$ and uncoupling the eNOS enzyme via oxidation of the essential cofactor BH4 (Fig. 2). ${ }^{10,11,21}$ Additionally, excessive production of ROS within the mitochondria diminishes the production of $\mathrm{NO}$ via eNOS uncoupling as well as quenching of $\mathrm{NO}$ and the production of peroxynitrite anion (ONOO-). These processes lead to decreased bioavailable retinal vascular $\mathrm{NO}$.

\section{CMS, Obesity and Insulin Resistance (IR)}

CMS comprises a cluster of cardiovascular risk factors including obesity, dyslipidemia, IR, hypertension, dysglycemia, albuminuria and diminished renal function. Recent data suggests that the prevalence of DR and vision-threatening DR is especially high in African Americans. ${ }^{2}$ While hyperglycemia has been thought to be the main predictor of microvascular complications in patients with T2DM, this risk may be modified by some of the components of the CMS. Additionally, persons with CMS in the Atherosclerosis Risk in Communities (ARIC) study were significantly more likely to have retinopathy than people without the syndrome, independent of age, gender, race, education, cigarette smoking and alcohol consumption. The association of the CMS with retinal microvascular remodeling, may be a way to evaluate the impact of these individual syndrome components and the presence of retinal lesions. ${ }^{2,3}$ Experimental studies have shown that Zucker diabetic fatty (ZDF) rats develop microvascular retinopathy with characteristic capillary basement membrane thickening. ${ }^{22}$

\section{Obesity and Diabetic Retinopathy}

The obesity epidemic is closely related to the CMS and T2DM epidemic and obesity contributes to DR. ${ }^{23-25} \mathrm{DR}$ is a multifactorial microvascular complication, involving glucose and lipid 


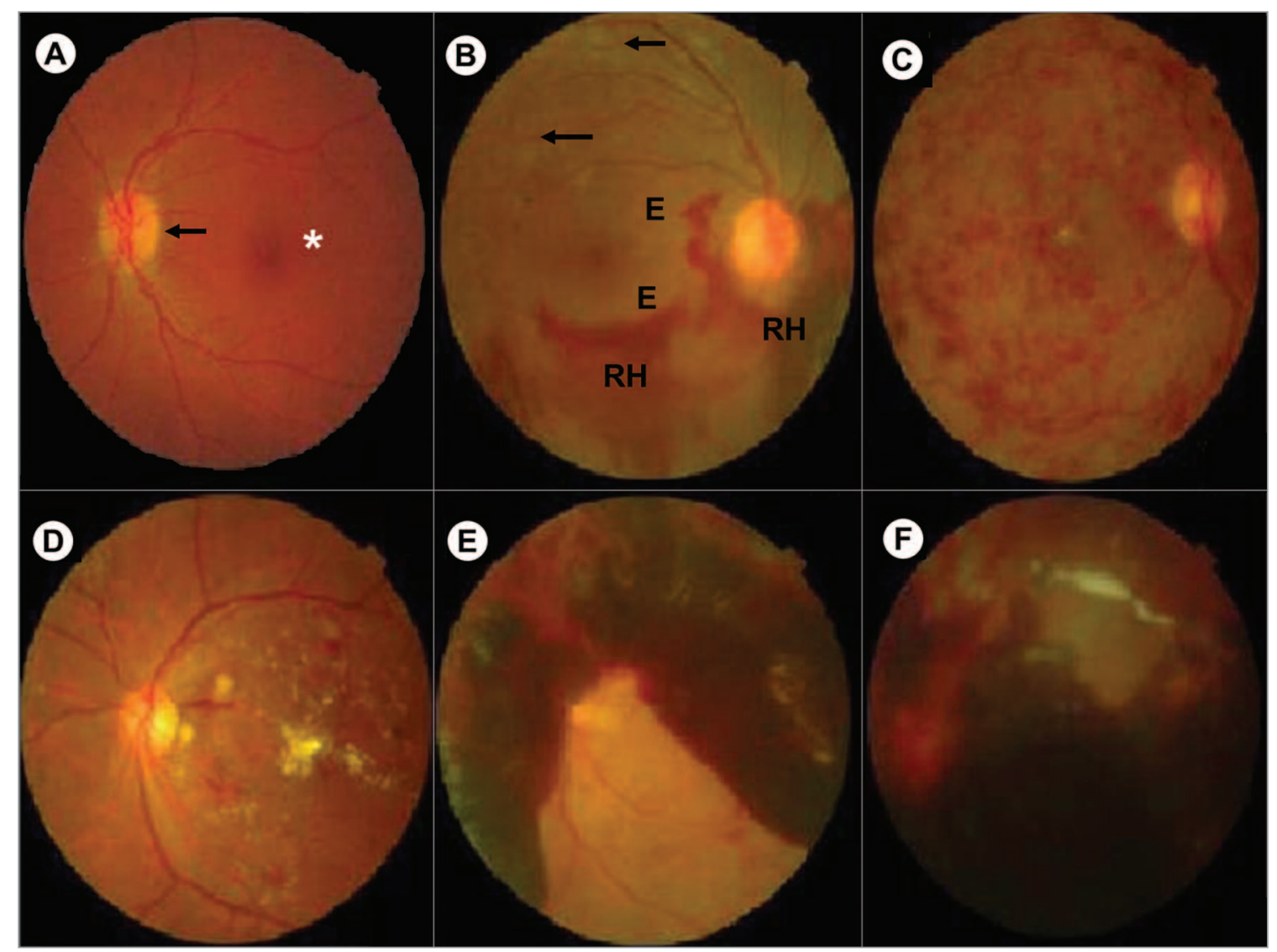

Figure 4. Characterization of Retina in Human Diabetic Retinopathy. (A) depicts the normal appearance of the retina. The macula is the darkened red spot in the center of the picture (asterisk). The yellow circle is the optic nerve (arrow). (B) displays dilated capillaries (microaneurysms), which leak red blood cells and plasma into the substance of the retina. This results in the appearance of retinal hemorrhages (RH), edema (E) and deposits or exudates (arrows). (C) demonstrates extensive intraretinal microvascular abnormalities, cotton wool spots, venous dilation and multiple extensive hemorrhages. (D) depicts macular edema with multiple hard exudates. (E) demonstrates vitreous hemorrhage as a result of neovascularization bleeding and hemorrhage. (F) depicts retina detachment and vitreous hemorrhage.

metabolism, blood pressure and BMI. Increased capillary permeability, microaneurysm formation, capillary closure and retinal ischemia are probably due to the combined effects of the various risk factors associated with retinal redox stress and remodeling. Obesity may increase oxidative stress because of its associated hyperleptinemia and high levels of plasma leptin are associated with DR. Further, decreased physical activity is a risk factor for $\mathrm{DR}^{2}$ Importantly, those with elevated BMI and other components of the CMS should be given more frequent ophthalmologic examinations. $^{23-25}$

\section{Glucotoxicity}

Hyperglycemia causes retinal reductive stress (pseudohypoxia) which contributes to the overall redox stress induced endothelial dysfunction - a process associated with vasoconstriction, thrombosis, inflammation and retinal remodeling. Observations in the type 1 diabetic rats suggest that long-term hyperglycemia is necessary for hyperglycemia to initiate changes to the retinal vasculature. This process involves increased ROS and glucose autoxidation and activation of the polyol, hexose flux, PKC and AGE pathways (Fig. 2).

\section{Hypertension}

The UKPDS demonstrated that intensive blood pressure control reduced the risk of DR in patients with T2DM independent of glycemic levels. The Australian Diabetes, Obesity and Lifestyle Study (Aus-Diab) further demonstrated that the two major risk factors of DR were hyperglycemia and hypertension. ${ }^{26}$ Hypertension is associated with increased angiotensin II (Ang II) and aldosterone and both are known to increase vascular $\mathrm{NAD}(\mathrm{P}) \mathrm{H}$ oxidase, which is associated with increased ROS generation. ${ }^{5,6,18}$ In diabetic microangiopathy, it was reported that $\mathrm{NAD}(\mathrm{P}) \mathrm{H}$ oxidase activity was increased in the retina of diabetic rats.

\section{Endothelial Dysfunction and Diabetic Retinopathy}

Endothelial dysfunction and damage is pervasive in the CMS (Fig. 6) plays a significant role in the development of T2DM and diabetic complications. DR is associated with the presence of microvascular vasculopathy and associated endothelial dysfunction. Accumulating evidence has demonstrated that 


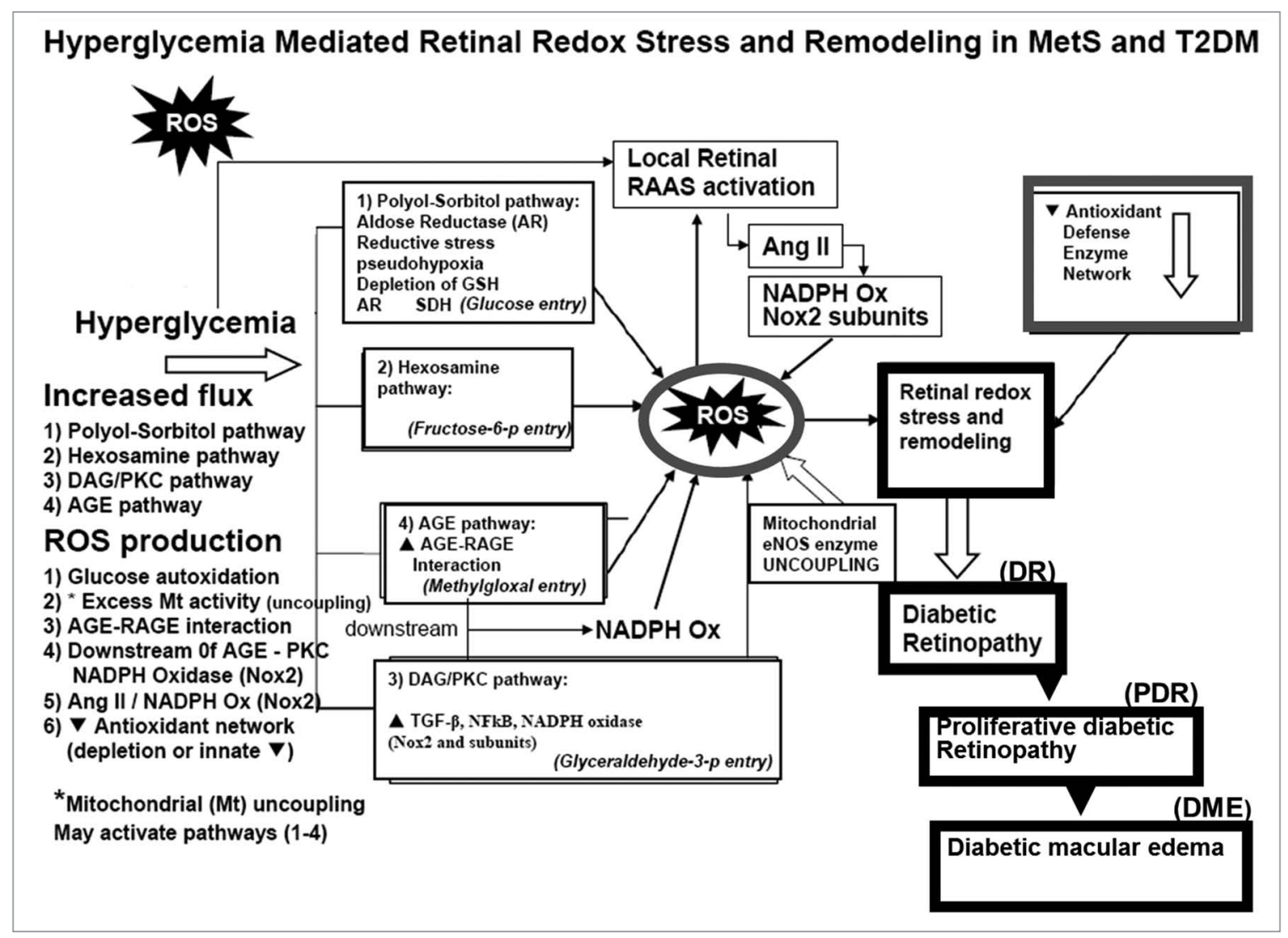

Figure 5. Schematic of the Hyperglycemia Mediated Retinal Redox Stress and Remodeling in Metabolic Syndrome and Type 2 Diabetes Mellitus. Note how hyperglycemia is important in activating the four major pathways involved in the production of excessive reactive oxygen species (ROS) to result in diabetic retinopathy (DR), eventual proliferative diabetic retinopathy (PDR) and diabetic macular edema (DME). AGE, advanced glycosylation endproducts; Ang II, angiotensin II; DAG/PKC, diacylglycerol protein kinase C; AR, aldose reductase; DME, diabetic macular edema; DR, diabetic retinopathy; eNOS, endothelial nitric oxide synthase; GSH, glutathione; Mt, mitochondria; NADPH, reduced nicotinamide adenine dinucleotidephosphate; NFKB, nuclear factor kappa B; Nox 2, NADPH Oxidase 2; RAAS, renin-angiotensin-aldosterone system; RAGE, receptor for advanced glycation endproducts; ROS, reactive oxygen species; TGF $\beta$, transforming growth factor beta.

hyperglycemia is directly implicated with endothelial dysfunction. ${ }^{10,27}$ ROS induced by hyperglycemia in EC plays a key role in the pathogenesis of DR (Fig. 5). When EC were exposed to increasing concentrations of glucose, there was an associated increased generation of ROS. ${ }^{10}$ The four previously discussed metabolic pathways have been shown to promote superoxide overproduction by the mitochondria electron-transport chain resulting in endothelial damage and dysfunction (Fig. 5). Excessive mitochondrial superoxide may induce mitochondrial DNA mutations resulting in persistent defective electron transport chain abnormalities and endothelial dysfunction.

\section{Redox Stress, Inflammation, Cytokines, Growth Factors and Diabetic Retinopathy}

Chronic inflammation may play an important role in the development and progression of DR. ROS are regarded as a strong stimulus for the release of cytokines and interleukin (IL)- $1 \beta$ itself can trigger signaling cascades resulting in excessive ROS. ${ }^{28}$ Cytokines are known to increase superoxide formation and can promote inflammation through various pathways, damage EC, increase microvascular permeability promoting the additional production of cytokines, and contribute to the recruitment of neutrophils, monocytes, macrophages and activate microglia at inflammatory sites of the retina. ${ }^{28,29}$

ROS are known to activate the nuclear transcription factor, $\mathrm{NF \kappa} \beta$ and this powerful nuclear activator is known to activate downstream inflammatory cytokines, $\mathrm{NO}$ and prostaglandins. The levels of cytokines including IL-1 $\beta$, IL- 6 and IL- 8 are increased in the vitreous fluid of patients with proliferative DR and in retinas of diabetic rodent models.

Activated PKC can bring about a variety of changes characteristic of DR. The action of several factors such as vascular endothelial growth factor (VEGF), pigment epithelium derived factor (PEDF), ${ }^{30}$ insulin-like growth factor 1 (IGF-1), ${ }^{31}$ transforming growth factor $\beta$ and adiponectin (ADPN). ${ }^{32}$ These proteins regulate the changes characteristic of DR including increased vessel permeability, blood flow, alteration of hormones and growth factor receptor recycling, stimulation of neovascularization, endothelial proliferation and apoptosis, which activate PKC.

Ultrastructural remodeling abnormalities result from a cascade of biochemical molecular functional signaling mechanisms 
involving ROS and tissue-cellular remodeling. ROS-redox stress, NFк $\beta$ and downstream cytokine activation, inflammation and the angiogenesis. ${ }^{33}$

\section{Vascular Endothelial Growth Factor (VEGF)}

VEGF has been established as a survival factor in the renal and retinal capillary endothelium. Redox stress and remodeling has been correlated with increased production of VEGF under in vitro conditions and is thought to be involved in the upregulation of VEGF expression during DR. ${ }^{16} \mathrm{DR}$ is accompanied by elevation in various angiogenic factors and VEGF is considered to play a pivotal role in the increased permeability and angiogenesis in this disease. VEGF is required for the development of diabetic retinal neovascularization and it can induce much of the pathology of DR. In a recent study proliferative DR patients had elevated plasma VEGF levels and increased VEGF-634C/G
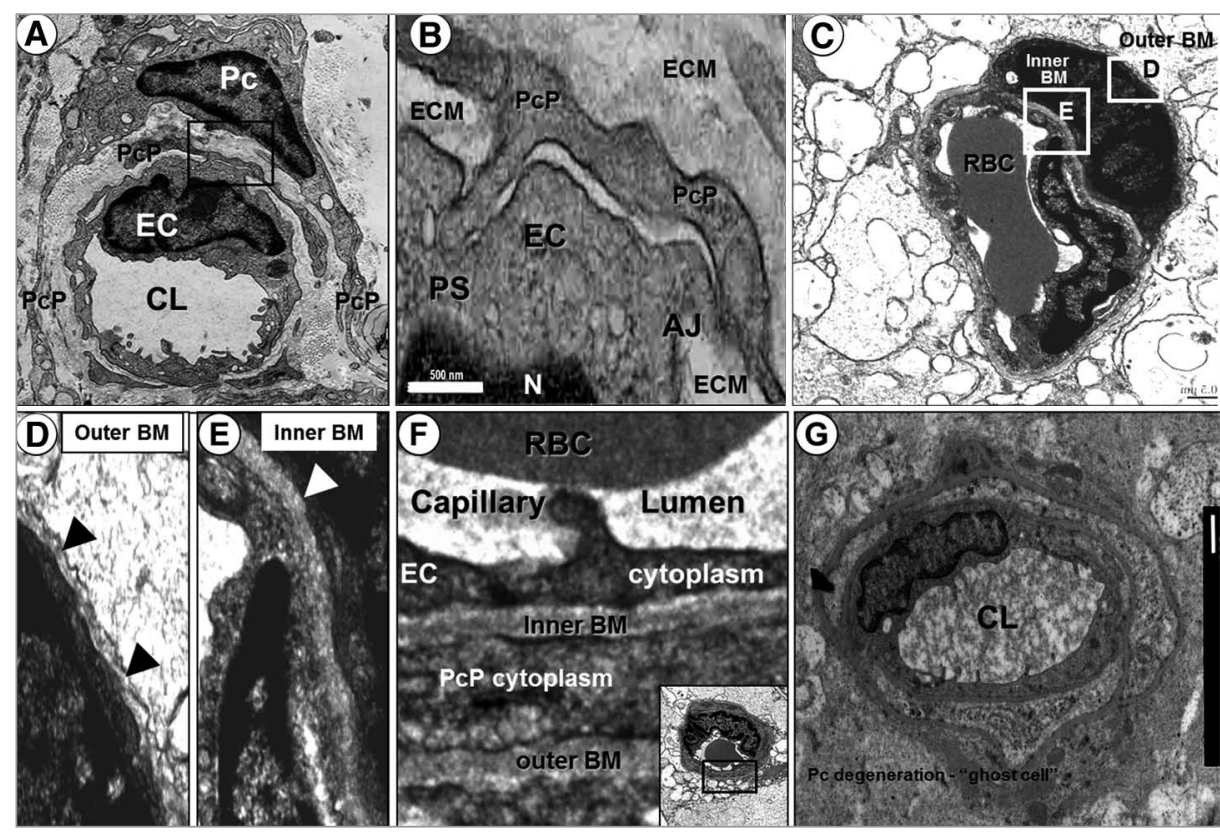

Figure 6. Morphological Differences Between Peripheral Continuous Capillaries and Blood-Retinal Barrier Capillaries. Peripheral continuous capillaries do not share a BM; rather, they communicate with peg sockets (PS) and adherens junctions (AJ) as in (A and B). In the blood-retinal barrier this communication is shared via a common inner and outer basement membrane (BM) with the pericyte being uniquely sandwiched or encapsulated by a prominent inner and outer BM.

polymorphism. ${ }^{34}$ In diabetic hyperglycemic rats, retina VEGF concentrations are increased, and this can be blocked by antioxidant treatment. ${ }^{35}$ Additionally, AGE increased VEGF expression in retinal pigment epithelial (RPE) and vascular smooth muscle cells (VSMC). Anti-VEGF antibody blocked capillary EC proliferation in AGE treated cells. ${ }^{16}$

\section{Pigmented Epithelial Derived Factor (PEDF)}

PEDF is a member of the serine protease inhibitor (serpin) superfamily with neurotrophic and antiangiogenic properties, and a decreased level of PEDF in the eye is important in the pathogenesis of proliferative DR. ${ }^{36} \mathrm{PEDF}$ is a natural extracellular component of the retina and has been found in the vitreous and aqueous humors.

Decreased levels of PEDF were reported in the ocular fluids of patients with angiogenic eye diseases. ${ }^{37} \mathrm{PEDF}$ has potent antiangiogenic activity in retinal EC growth and migration and suppressed ischemia-induced retinal neovascularization. ${ }^{38}$

Pericyte loss is one of the earliest hallmarks of diabetic retinopathy and an important reason for pericyte loss is ROS. ${ }^{30} \mathrm{In}$ DR, PEDF has a novel benefit since PEDF protects retinal pericytes against oxidative stress-induced injury through its anti-oxidative properties, which might slow the development of diabetic retinopathy. ${ }^{30}$

PEDF protects against high glucose or ROS induced pericyte apoptosis and dysfunction through its anti-oxidative properties via induction of glutathione. ${ }^{39}$

Additionally, PEDF induces the ERK signal cascade which contributes to retinal pigment epithelial cell cytoprotection against oxidative stress. ${ }^{40}$ Thus, retinal cells including the BRB capillaries and their supportive and protective pericytes may possess a system capable of efficiently responding to PEDF. ${ }^{39-41}$

\section{Adiponectin (ADPN)}

The adipocyte derived factor ADPN is an insulin sensitivity activator, and is correlated to retinal redox stress and remodeling in metabolic syndrome and T2DM. Low levels of serum ADPN levels were found to be correlated with the severity of retinopathy. ${ }^{32}$ Insulin-sensitizing agents reduce pathological retinal microvessel formation through ADPN mediated modulation of tumor necrosis factor alpha (TNF $\alpha$ ) production. ${ }^{42}$ ADPN's effect on diabetic retinopathy is not clear. However, ADPN induces eNO production by stimulating phosphorylation and activation of eNOS. ADPN inhibits specific binding of oxidized LDL and its uptake by macrophages..$^{41} \mathrm{ADPN}$ possesses anti-inflammatory properties and thus may negatively modulate the process of atherogenesis. ${ }^{43,44}$ ADPN suppresses adverse effects of inflammatory cytokines and reduces oxidative stress induced by oxidized LDL or high glucose in EC. ${ }^{44-48}$ ADPN inhibits VEGF-stimulated human coronary artery EC migration via cAMP/PKA dependent signaling including VEGF-induced generation of ROS, which implicates it as an important role in vascular processes associated with diabetes. Because ADPN is known to act as an antioxidant, anti-inflammatory, antiapoptotic and antifibrotic protein then its low levels may predispose it to a loss of any or all of the above known protective features of ADPN and directly or indirectly affect the capillary BRB including the pericyte. Importantly, ADPN may be used in the future as an early candidate biomarker of DR in CMS and T2DM. 


\section{Insulin-like Growth Factor-1 (IGF-1)}

Retinal IGF-1 mRNA levels are lower in the human and diabetic rat when compared to age matched non-diabetic controls $s^{49}$ and IGF-1 can have direct mitogenic effects on retinal EC. ${ }^{31}$ IGF-1 can stimulate glucose transport into retinal microvascular $\mathrm{EC}$ via activation of $\mathrm{PKC}$ and can modulate the expression and activity of VEGF. ${ }^{31}$ Similar to VEGF, the activation of IGF-1 also increases PKC activation, ${ }^{50}$ so IGF-1 may be regulated by oxidative stress via the PKC pathway. ${ }^{51}$

\section{Transforming Growth Factor $\beta$ (TGF $\beta$ )}

TGF $\beta$ is an important factor mediating BM thickening and matrix accumulation that are the hallmark of diabetes in blood vessels and vascular structures. ${ }^{36}$ Most of these results are from renal research. TGF $\beta$ signaling is increasing in early diabetic retinal vessels of rat $^{37}$ and the aldose reductase inhibition normalizes expression of TGF $\beta$. Thus, TGF $\beta$ is an important inflammatory cytokine in DR and closely related with excessive ROS and eventually fibrosis. ${ }^{18}$

Diabetic retinopathy is regarded as a low level chronic inflammatory disease and one of the triggers of cytokine release is excessive ROS. ${ }^{38}$ The levels of cytokines, including IL-1 $\beta$, IL- 6 , IL-8 and $\mathrm{TNF} \alpha$ are increased in the vitreous of patients with proliferative diabetic retinopathy and in the retina from diabetic rats and mice. ${ }^{39}$

\section{Interleukin-1 Beta (IL-1 $\beta)$}

IL-1 $\beta$ gene expression is known to reside in EC and glial cells and its expression is significantly upregulated in high glucose conditions allowing for BRB allowing inflammatory cells to increase their migration across the BRB. ${ }^{52}$ Additionally, IL- $1 \beta$ is known to increase the expression of VEGF in retinal EC, and induces the expression of various genes whose promoters are regulated through complex interactions with $N F \kappa \beta .{ }^{28}$ IL-1 $\beta$ has been found to be increased in streptozotocin diabetic rat model $s^{52}$ and IL-1 $\beta$ accelerates apoptosis in retinal capillary cells, specifically pericytes, through activation of $N F \kappa \beta$, which is exacerbated by high glucose conditions. ${ }^{53,54} \mathrm{NF \kappa} \beta$ is a key regulator of antioxidant enzymes and can initiate transcription of genes involved in apoptosis and additionally increases downstream inflammatory cytokines. ${ }^{40,54}$ Importantly, IL-1 $\beta$ activation-stimulation results in the translocation of NFK $\beta$ from its cytosolic compartment to the nucleus where it initiates apoptotic genes and downstream inflammatory cytokines..$^{53}$ Additionally, IL-1 $\beta$ is considered as one of the most potent stimuli for inducible NOS (iNOS), contributing to ongoing inflammation via induction of iNOS protein and augmentation of its activity. ${ }^{52} \mathrm{IL}-1 \beta$ receptor antagonism (IL-1 $\beta \mathrm{ra}$ ) in the retina and recently IL-1ßra have been shown to interfere with the development of not only diabetic retinopathy but also pancreatic islet inflammation and beta cell apoptosis in humans with T2DM. ${ }^{52,55}$

\section{Interleukin-6 (IL-6)}

The IL- 6 cytokine shares common characteristics with VEGF, in that both are induced by hypoxia and hyperglycemia, and both play a role in vascular inflammation, permeability and angiogenesis. ${ }^{41}$ Recent human studies have demonstrated that both VEGF and IL-6 were elevated in aqueous humor of patients with DR and even higher in those with proliferative DR indicating that VEGF and IL-6 play important roles in the development of DR. ${ }^{56}$ Even peripheral blood levels of IL-6 and TNF $\alpha$ were elevated in humans with DR with the highest elevations found in those with proliferative DR.$^{57}$ It has been shown that the Ang II-induced vascular alterations involved activation of NAD(P) $\mathrm{H}$ oxidase, IL-6, and increases in VEGF expression and further, that deletion of IL- 6 prevented these effects of vascular inflammation in DR. ${ }^{58}$

\section{Monocyte Chemoattractant Protein (MCP-1)}

MCP-1 contributes to the recruitment of inflammatory cells (monocytes/monocyte derived macrophage/microglia) in injured tissue and ROS injury may be in play in DR and retinal detachment. ${ }^{59} \mathrm{MCP}-1$ is a potential angiogenic factor in the proliferative phase of DR and is associated with proliferation DR. ${ }^{42}$ Hyperglycemia increases the expression of MCP-1 in vascular $\mathrm{EC}^{43}$ and AGE-induced ROS generation induced the MCP-1 gene and mRNA expression. ${ }^{44,45}$ Recently, aqueous samples in humans with DR have revealed higher levels of MCP-1 and VEGF when compared to nondiabetic subjects and authors further state that inflammatory changes may precede the development of neovascularization in proliferative $\mathrm{DR} .{ }^{60}$

\section{Nuclear Factor KappaBeta $\left(\mathrm{NF}_{\kappa} \beta\right)$}

NFк $\beta$ has been known for some time to be redox sensitive and activated by oxidative-redox stress and ROS. ${ }^{54,61}$ Further, it is known that glucotoxicity via autoxidation, mitochondrial leakage, activation of $\mathrm{NAD}(\mathrm{P}) \mathrm{H}$ oxidase and AGE/RAGE interaction promote a heightened redox state in cells in patients with diabetes. ${ }^{5,6,11,18}$ Additionally it is known that $\mathrm{NF} \kappa \beta$ plays a pivotal role in atherosclerosis, ${ }^{5,6,11}$ which is accelerated in the CMS and T2DM. NFK $\beta$ promotes activation of downstream proinflammatory cytokines, upregulation of endothelin and apoptosis. Interestingly, $\mathrm{NF \kappa} \beta$ promotes apoptosis in pericytes and not in ECs both in vitro and in vivo situations. ${ }^{54}$ It is quite interesting that once NFK $\beta$ is activated, it in turn is responsible for the activation of downstream cytokines, which promotes a further increase of ROS and once again the nuclear transcription factor is activated promoting a viscous cycle of ROS-NFк $\beta$-ROS, which results in cell injury promoting apoptosis.

\section{Novel Choroid and Plexiform Blood Retinal Barrier (BRB) Capillary Remodeling in Young Zucker and Diabetic Porcine Models}

Ultrastructural remodeling in retinas of young models of the CMS-IR (Obese fa/fa Zucker rat model) and early overt diabetes ( 20 week old alloxan diabetic porcine models) were utilized for this brief review. Qualitative remodeling of the BRB in the microvascular choroid coat layer and the less vascular optic nerve 

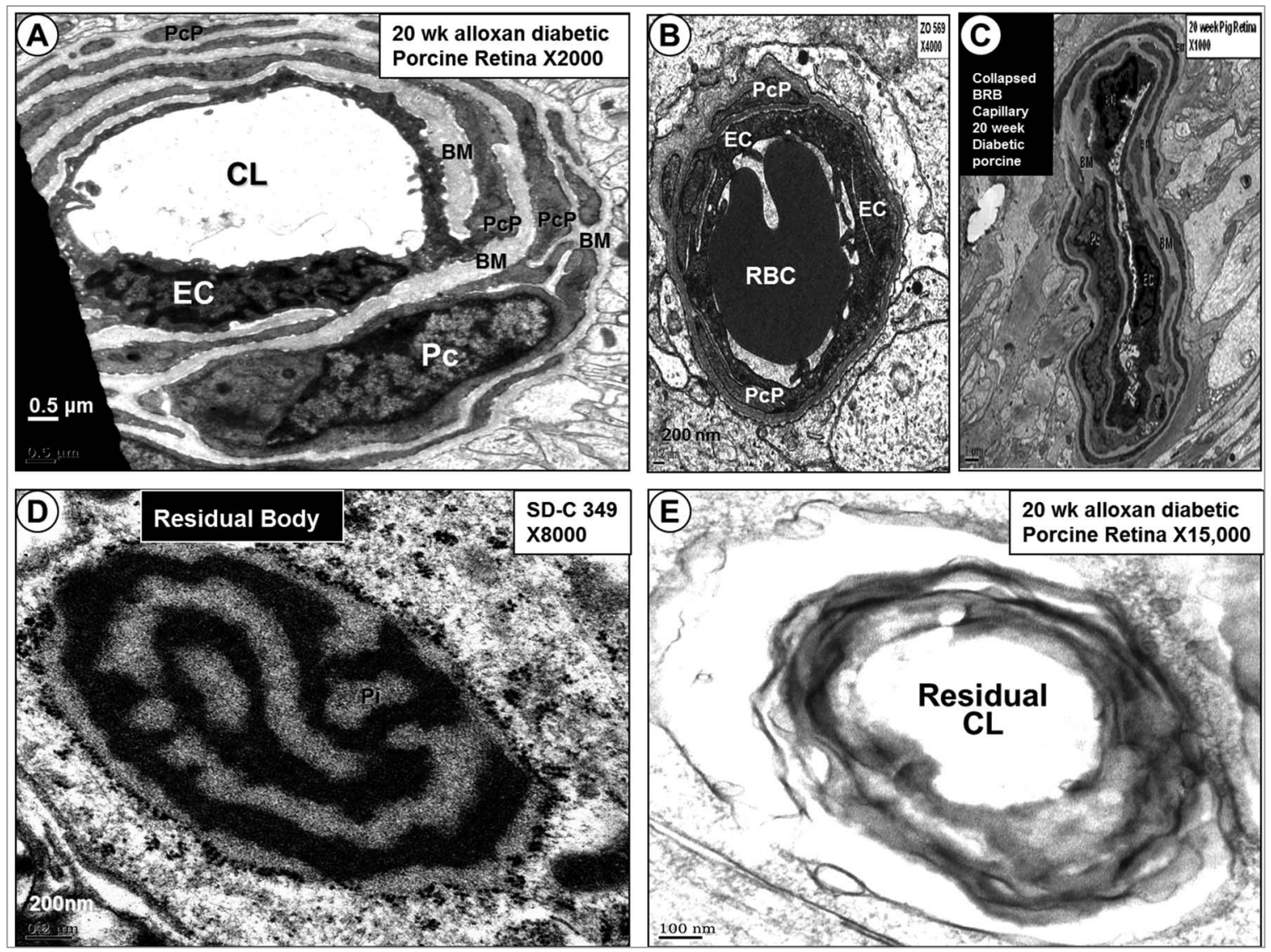

Figure 7. Fate of 20 Week Old Alloxan Diabetic Retinal-Blood Barrier Capillaries. (A) depicts the pericyte process remodeling and the thickened basement membranes (BM) found in the remaining and surviving retinal-blood barrier capillaries. Note the numerous enveloping-encircling pericyte processes (PcP) as it protects the surviving endothelial cell (EC) and it's thickened continuous BM. (B and C) illustrate capillary collapse of retinal-blood barrier capillaries in the 20 week alloxan diabetic porcine capillaries as compared to representative 9 week old Zucker lean and obese models. (D) illustrates illustrate a typical residual body and this was found in a podocyte of the 9 week old Zucker Obese kidney. (E) depicts a similar appearing residual body of what is thought to be a residual body of a demised-degenerated retinal-blood barrier capillary found in the 20 week alloxan diabetic porcine model.

fiber and plexiform layers beneath the inner limiting membrane of the retina are presented (Fig. 1).

Findings in the young Zucker models did not reveal any marked differences in BM thickness or pericyte morphology and BRB capillaries were abundant in both the Zucker falfa obese and Zucker lean control models. Observations did allow the microscopist's to observe that the unique BRB pericytes were doubly encased by a BM and pericytes were sandwiched between an inner and outer impervious prominent BM being surrounded by an electron dense reticular extracellular matrix (Figs. 2, 3 and 6). In contrast to the young Zucker model's, the 20 week alloxan diabetic porcine model revealed the following qualitative changes: marked reduction in the total number of BRB capillaries (capillary rarefaction), capillary collapse (Fig. 7C), pericyte degenerative changes consisting of ghost cells with loss of cytoplasmic matrix electron density with apoptosis and formation of residual bodies (Fig. 7E). The remaining surviving capillaries displayed pericytes that encircled the surviving EC multiple times (Fig. 7A). Multiple layers of BMs and pericyte processes encircling the surviving EC may be a structural remodeling attempt to protect the omnipotent EC from multiple metabolic toxicities associated with obesity, CMS, prediabetes and overt T2DM. Indeed, it is the duration of these metabolic toxicities and the subsequent cellular signaling dysfunctions that predispose to end-organ damage as occurs in the retinal microvascular tissues. The unique structure of the doubly encased sandwiched pericyte by and inner and outer BM and the ECM contribute to these microvascular changes in DR. Further studies should access each of these changes observed in the porcine model to see if they occur at an earlier stage in human DR as well as the ultrastructural endothelial tight junctions in the Zucker and porcine models.

\section{Conclusion}

The central theme for previously published remodeling in CMS and T2DM has been the multiple metabolic toxicities (A-FLIGHT-U) acting singularly and synergistically to produce excessive and damaging ROS.,18 These ROS may arise from either oxidation or reduction and therefore the term redox stress is utilized in addition to the more common and classical term of oxidative stress. This brief review of DR is based on 


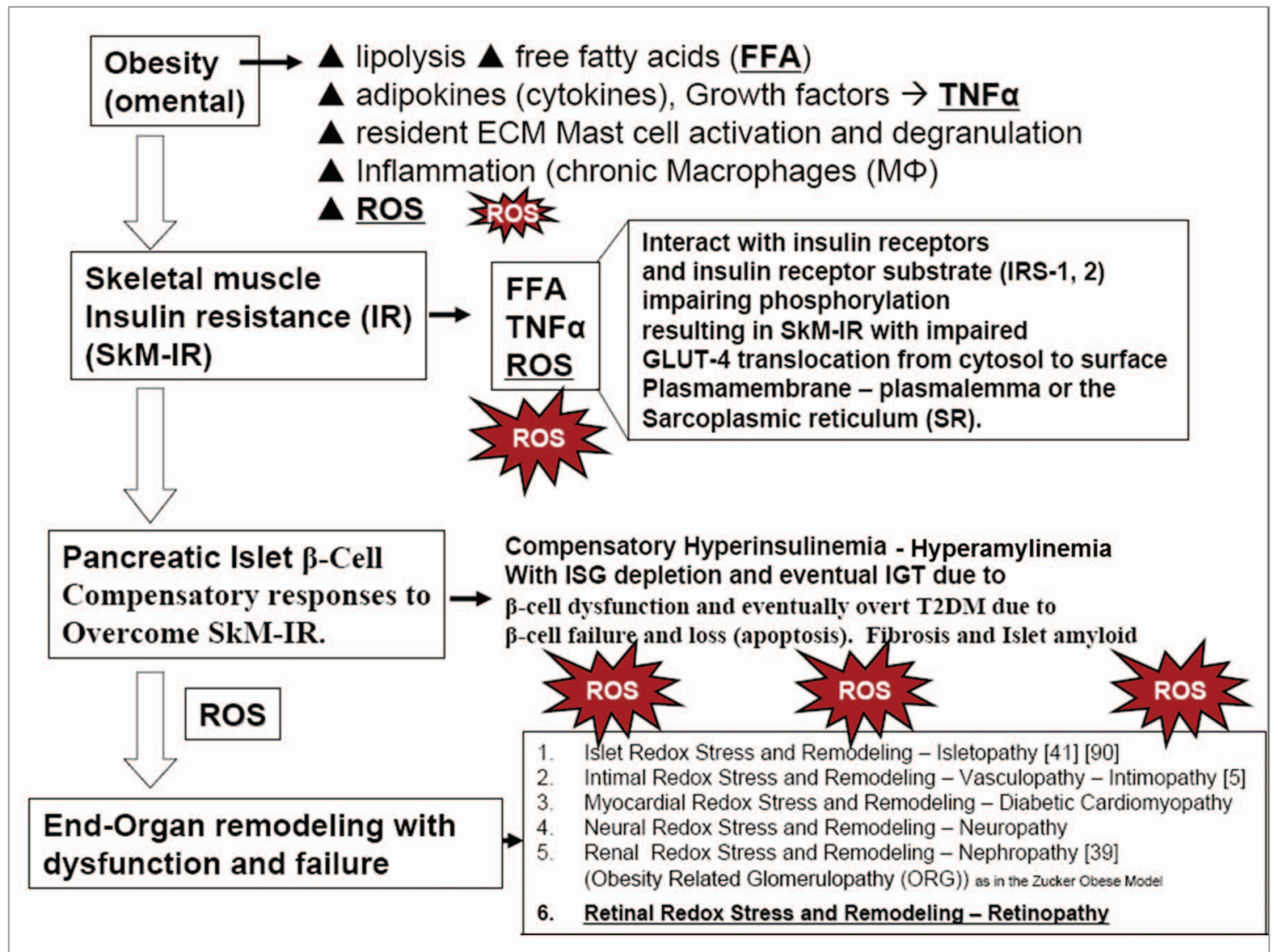

Figure 8. Sequence of Events in the Development of Metabolic Syndrome and overt Type 2 Diabetes Mellitus. This figure illustrates the sequence of events leading to end-organ remodeling with dysfunction and eventual failure and begins with obesity and subsequent skeletal muscle insulin resistance (IR) involving free fatty acids (FFA), tumor necrosis factor alpha (TNF $\alpha$ ) and reactive oxygen species (ROS). This is followed by a compensatory islet beta-cell ( $\beta$-cell) response in order to overcome skeletal muscle IR and is associated with eventual $\beta$-cell dysfunction and failure resulting in type 2 diabetes mellitus (T2DM). Additionally, there is glucotoxicity once impaired fasting glucose-prediabetes and overt T2DM develops contributing to additional ROS in addition to the ROS generated by the A-FLIGHT-U multiple metabolic toxicities and the ensuing end-organ damage resulting in the multiple end-organ failure and multiple diabetic pathologies including diabetic retinopathy.

translational research designed to further delay or prevent the abnormal structural remodeling associated with these diabetic end-organ pathologies..$^{1-5}$ Importantly, DR and the other diabetic end-organ pathologies (isletopathy, cardiomyopathy, neuropathy and nephropathy) are intimately associated with injury to the microvessel milieu. Redox stress; cellular-extracellular injuries result in structural remodeling and dysfunction with the eventual development of pathologies and end-organ failure (Fig. 8)..$^{1-5}$

In this brief review, we have tried to explain the relationship between retinal redox stress and remodeling in CMS and T2DM in DR. The traditional and well accepted diabetic retinopathy mechanisms, including increased polyol pathway, activation of PKC, increased AGE, and increased hexosamine pathway are driven largely through redox stress and ROS. This unifying mechanism of retinal redox stress and remodeling appears to be playing a significant role at each step in the development of T2DM and DR, ROS induce the nuclear transcription factor $N F \kappa \beta$ with subsequent activation of numerous downstream cytokines. Additionally, the growth factors IGF-1 and TGF $\beta$ are critically important in redox stress and the excessive production of ROS.
Since retinal redox stress and remodeling occur not only in type 1 diabetes mellitus, but also in T2DM and in CMS, antioxidant therapy either direct or indirect may have potential for delaying or preventing the progression of DR and its treatment. It has been reported that some traditional antioxidants have been tried, such as vitamin $\mathrm{C}$, vitamin $\mathrm{E}$, lipoic acid, antioxidative enzymes, taurine, acetylcysteine, and have some positive effect on DR. ${ }^{15,45,51}$ Supplementation of vitamins $\mathrm{C}$ and $\mathrm{E}$ for 18 months inhibited retinopathy, ${ }^{45}$ and supplementation of vitamins $\mathrm{C}$ and $\mathrm{E}$ for two months in rat models have helped alleviate the subnormal activity of antioxidant defense enzymes. The antioxidants, ascorbic acid $(1.0 \%)$ plus alpha-tocopherol $(0.1 \%)$ inhibited the decrease of gamma-glutamyl transpeptidase activity and glutathione levels in rat model retinas. ${ }^{15}$

Supplemental dietary ascorbic acid and alpha-tocopherol acetate for two months had a partial beneficial effect on the activity of retinal $\mathrm{Na}^{+}$and $\mathrm{K}^{+}$-ATPase in diabetic and galactosemic rats. ${ }^{46}$ However, lipoic acid did not improve the impaired retinal blood flow comparable to high doses of vitamin E. Antioxidant treatments including vitamin $\mathrm{C}$, vitamin $\mathrm{E},{ }^{47}$ and lipoic acid may 
provide positive effects in DR. ${ }^{48}$ Antioxidant therapy has limited utility in some organs, because scavenging of preformed unpaired electrons/ROS after damage to tissues has already occurred and may be utilized too late to result in any clinical improvement. Another reason for limitation is the dose utilized in clinical studies is not as concentrated as in vitro and animal studies. Antioxidant therapies are directed towards not only increased generation of ROS but may also allow for the possibility of the naturally occurring antioxidant defense network to be restored and improve the overall ROS scavenging mechanisms.

Nicanartine (5-(3,5-di-tert-butyl)-4-hydroxyphenyl-1-(-pyridyl)2-oxapentane CAS 150443-71-3, Mrz 3/124), an antioxidant and antiproliferative agent which reduces lipid peroxidation, partially inhibited pericyte loss, but failed to have any effect on the number of acellular capillaries or microaneurysms in diabetic rats. Diabetic rats supplemented with the water-soluble antioxidant Trolox for five months partially restored diabetes induced change in the ratio of retinal capillary EC to pericytes. ${ }^{49}$

PEDF decreases redox stress in pericytes and might be a potential therapeutic strategy for treatment of patients with early diabetic retinopathy. ${ }^{49}$ Curcumin, a compound with antioxidant and anti-inflammatory properties, administration inhibited IL-18, induced an increase in VEGF production, and prevented diabetes-induced increased IL-1 $\beta .^{32}$ IGF-1 therapy may also be a candidate therapy as it can improve insulin sensitivity; even though, recombinant human IGF-1 has an adverse effect in obese-IR-T2DM patients. ${ }^{50}$ Future therapeutic application of these treatments in human medicine will require further study at this time.

In summary, this brief review has examined ultrastructure remodeling in two different animal models (the young Zucker and an alloxan diabetic porcine model). Some novel findings emerged that might help to better understand certain BRB remodeling characteristics associated with DR. Both the ECM and the morphology of BRB pericytes displayed a unique matrix remodeling which may contribute to the early BRB remodeling in the retina. The more electron dense and tightly packed ECM surrounding the optic nerve fiber layer, plexiform layers and the choroid coat could have a bearing on morphological remodeling as well as functional changes.

In addition to the ECM differences, the pericytes in the BRB are doubly encased and sandwiched between two rather prominent BMs unlike other peripheral capillaries in the body.
Thus, the pericyte is more impermeable to phagocytes when they are undergoing degeneration due to the metabolic toxicities and excessive redox stress. ${ }^{62}$ This creates a milieu for the degenerative products of necrosis - apoptosis, which may allow for the early BM thickening. Focusing future research based on matrix and morphologic uniqueness in the BRB may help to unlock new ideas in treatment of DR and its complications. A better understanding of redox homeostasis and retinal redox stress and remodeling may enable each of us to develop a shift in our paradigm to an earlier intervention during the CMS and pre-diabetes phase to delay or prevent the development of DR.

While this brief review specifically examined the longevity of the pericyte, researchers and clinicians should consider preserving the longevity of all cells, tissues and organs in the process of treating chronic metabolic excesses and cellular injury. ${ }^{63}$

In recent years there has been accelerated and mounting evidence regarding the importance of injury due to redox stressROS, hypoxia and ischemia-reperfusion injury followed by the natural occurring wound healing process in tissues and organs. If the injurious stimuli is chronic, as occurs in chronic metabolic excesses associated with the CMS and T2DM, this natural wound healing mechanism will also become chronic. This will allow persistent chronic inflammation, inflammatory cells, growth factors, angiogenesis and fibrosis in the pathogenesis not only of diabetic retinopathy but also most aging-related chronic diseases, which will result in a myriad of diseases that will increasingly affect the multitude of the aging baby boom generation. If researchers and clinicians can collectively break this cascade of ROS begetting ROS, inflammation, cytokine-growth factor, excessive angiogenesis and fibrosis cycle then we may be able to halt the natural progressive history of most chronic diseases affecting this rapidly aging population and the epidemic of obesity, CMS and T2DM in our childhood-adolescent youth with overweight and obesity. ${ }^{63}$

\section{Acknowledgements}

Authors acknowledge Dr. Martin Katz, Ph.D., director of the electron microscopy core facility and Cheryl Jensen (University of Missouri, Columbia, Missouri) for preparing retinal tissue samples for study and Brenda Hunter for her assistance in preparing this manuscript. Support for this research was provided for Y.Y., by a grant from the National Natural Science Foundation of China (No 30960148).

\section{References}

1. Zhang X, Saaddine JB, Chou C, Cotch MF, Cheng YJ, Geiss LS, et al. Prevalence of diabetic retinopathy in the United States 2005-2008. JAMA 2010; 304:649-56.

2. Wong TY, Duncan BB, Golden SH, Klein R, Couper DJ, Klein BE, et al. Associations between the metabolic syndrome and retinal microvascular signs: the Atherosclerosis Risk In Communities study. Invest Ophthalmol Vis Sci 2004; 45:2949-54.

3. Tikellis G, Anuradha S, Klein R, Wong TY. Association between physical activity measured during leisure activity and retinal microvascular signs: the Atherosclerosis Risk in Communities (ARIC) Study. Microcirculation 2010; 17:381-93.

4. Serne EH, Ijzerman RG, de Jongh RT, Stehouwer CD. Blood pressure and insulin resistance: role for microvascular function? Cardiovasc Res 2002; 53:271-6.
5. Hayden MR, Tyagi SC. Intimal redox stress: accelerated atherosclerosis in metabolic syndrome and type 2 diabetes mellitus. Atheroscleropathy. Cardiovasc Diabetol 2002; 1:3.

6. DeMarco V, Johnson MS, Whaley-Connell AT, Sowers JR. Cytokine abnormalities in the etiology of the cardiometabolic syndrome. Curr Hyperten Rep 2010; 12:93-8.

7. Gardner TW, Sander B, Larsen ML, Kunselman A, Tenhave T, Lund-Andersen $\mathrm{H}$, et al. An extension of the Early Treatment Diabetic Retinopathy Study (ETDRS) system for grading of diabetic macular edema in the Astemizole Retinopathy Trial. Curr Eye Res 2006; 31:535-47.

8. Wong TY, Klein R, Islam FM, Cotch MF, Folsom AR, Klein BE, et al. Diabetic retinopathy in a multi-ethnic cohort in the United States. Am J Ophthalmol 2006; 141:446-55.
9. Lee AY, Chung SS. Contributions of polyol pathway to oxidative stress in diabetic cataract. FASEB J 1999; 13:23-30.

10. Brownlee M. Biochemistry and molecular cell biology of diabetic complications. Nature 2001; 414:813-20.

11. Brownlee M. The pathobiology of diabetic complications: a unifying mechanism. Diabetes 2005; 54:1615-25.

12. Aiello LP, Gardner TW, King GL, Blankenship G, Cavallerano JD, Ferris FL, 3rd, et al. Diabetic retinopathy. Diabetes Care 1998; 21:143-56.

13. Miwa K, Nakamura J, Hamada Y, Naruse K, Nakashima E, Kato K, et al. The role of polyol pathway in glucose-induced apoptosis of cultured retinal pericytes. Diabetes Res Clin Pract 2003; 60:1-9.

14. Stitt AW. The role of advanced glycation in the pathogenesis of diabetic retinopathy. Exp Mol Pathol 2003; 75:95-108. 
15. Kowluru R, Kern TS, Engerman RL. Abnormalities of retinal metabolism in diabetes or galactosemia. II. Comparison of gamma-glutamyl transpeptidase in retina and cerebral cortex and effects of antioxidant therapy. Curr Eye Res 1994; 13:891-6.

16. Lu M, Kuroki M, Amano S, Tolentino M, Keough K, Kim I, et al. Advanced glycation end products increase retinal vascular endothelial growth factor expression. J Clin Invest 1998; 101:1219-24.

17. van Reyk DM, Gillies MC, Davies MJ. The retina: oxidative stress and diabetes. Redox Rep 2003; 8:187-92.

18. Hayden MR, Whaley-Connell A, Sowers JR. Renal redox stress and remodeling in metabolic syndrome, type 2 diabetes mellitus and diabetic nephropathy: paying homage to the podocyte. Am J Nephrol 2005. 25:553-69.

19. Ejaz S, Chekarova I, Ejaz A, Sohail A, Lim CW. Importance of pericytes and mechanisms of pericyte loss during diabetes retinopathy. Diabetes Obes Metab 2008; 10:53-63.

20. Murata M, Ohta N, Fujisawa S, Tsai JY, Sato S, Akagi $\mathrm{Y}$, et al. Selective pericyte degeneration in the retinal capillaries of galactose-fed dogs results from apoptosis linked to aldose reductase-catalyzed galactitol accumulation. J Diabetes Complications 2002; 16:363-70.

21. Yan SD, Schmidt AM, Anderson GM, Zhang J, Brett J, Zou YS, et al. Enhanced cellular oxidant stress by the interaction of advanced glycation end products with their receptors/binding proteins. J Biol Chem 1994; 269:9889-97.

22. Yang YS, Danis RP, Peterson RG, Dolan PL, Wu YO. Acarbose partially inhibits microvascular retinopathy in the Zucker Diabetic Fatty rat (SDF/Gmi-fa). J Ocul Pharmacol Ther 2000; 16:471-9.

23. van Leiden HA, Dekker JM, Moll AC, Nijpels G, Heine RJ, Bouter LM, et al. Risk factors for incident retinopathy in a diabetic and nondiabetic population: the Hoorn study. Arch Ophthalmol 2003; 121:245-51.

24. Henricsson M, Nystrom L, Blohme G, Ostman J, Kullberg C, Svensson M, et al. The incidence of retinopathy 10 years after diagnosis in young adult people with diabetes: results from the nationwide populationbased Diabetes Incidence Study in Sweden (DISS). Diabetes Care 2003; 26:349-54.

25. Zhang L, Krzentowski G, Albert A, Lefebvre PJ. Risk of developing retinopathy in Diabetes Control and Complications Trial type 1 diabetic patients with good or poor metabolic control. Diabetes Care 2001; 24:1275-9.

26. Tapp RJ, Shaw JE, Harper CA, de Courten MP, Balkau B, McCarty DJ, et al. The prevalence of and factors associated with diabetic retinopathy in the Australian population. Diabetes Care 2003; 26:1731-7.

27. Kawano H, Motoyama T, Hirashima O, Hirai N, Miyao Y, Sakamoto T, et al. Hyperglycemia rapidly suppresses flow-mediated endothelium-dependent vasodilation of brachial artery. J Am Coll Cardiol 1999; 34:146-54.

28. Fan F, Stoeltzing O, Liu W, McCarty MF, Jung YD, Reinmuth N, et al. Interleukin-1beta regulates angiopoietin-1 expression in human endothelial cells. Cancer Res 2004; 64:3186-90.

29. Ibrahim AS, El-Shishtawy MM, Peria A Jr, Liou GI. Genistein attenuates retinal inflammation associated with diabetes by targeting of microglial activation. Mol Vis 2010; 16:2033-42.

30. Amano S, Yamagishi S, Inagaki Y, Nakamura K, Takeuchi M, Inoue $\mathrm{H}$, et al. Pigment epitheliumderived factor inhibits oxidative stress-induced apoptosis and dysfunction of cultured retinal pericytes. Microvasc Res 2005; 69:45-55.

31. DeBosch BJ, Baur E, Deo BK, Hiraoka M, Kumagai AK. Effects of insulin-like growth factor-1 on retinal endothelial cell glucose transport and proliferation. J Neurochem 2001; 77:1157-67.
32. Kato K, Osawa H, Ochi M, Kusunoki Y, Ebisui O, Ohno K, et al. Serum total and high molecular weight adiponectin levels are correlated with the severity of diabetic retinopathy and nephropathy. Clin Endocrinol (Oxf) 2008; 68:442-9.

33. Ozturk BT, Bozkurt B, Kerimoglu H, Okka M, Kamis U, Gunduz K. Effect of serum cytokines and VEGF levels on diabetic retinopathy and macular thickness. Mol Vis 2009; 15:1906-14.

34. Yang Y, Andresen BT, Yang K, Zhang Y, Li X, Wang H. Association of vascular endothelial growth factor-634C/G polymorphism and diabetic retinopathy in type 2 diabetic Han Chinese. Exp Biol Med (Maywood) 2010; 235:1204-11.

35. Obrosova IG, Minchenko AG, Marinescu V, Fathallah L, Kennedy A, Stockert CM, et al. Antioxidants attenuate early upregulation of retinal vascular endothelial growth factor in streptozotocin-diabetic rats. Diabetologia 2001; 44:1102-10.

36. Becerra SP. Structure-function studies on PEDF. A noninhibitory serpin with neurotrophic activity. Adv Exp Med Biol 1997; 425:223-37.

37. Spranger J, Osterhoff M, Reimann M, Mohlig M, Ristow M, Francis MK, et al. Loss of the antiangiogenic pigment epithelium-derived factor in patients with angiogenic eye disease. Diabetes 2001; 50:2641-5.

38. Duh EJ, Yang HS, Suzuma I, Miyagi M, Youngman E, Mori K, et al. Pigment epithelium-derived factor suppresses ischemia-induced retinal neovascularization and VEGF-induced migration and growth. Invest Ophthalmol Vis Sci 2002; 43:821-9.

39. Yamagishi $S$, Inagaki $Y$, Amano S, Okamoto $T$, Takeuchi M, Makita Z. Pigment epithelium-derived factor protects cultured retinal pericytes from advanced glycation end product-induced injury through its antioxidative properties. Biochem Biophys Res Commun 2002; 296:877-82.

40. Tsao YP, Ho TC, Chen SL, Cheng HC. Pigment epithelium-derived factor inhibits oxidative stress-induced cell death by activation of extracellular signal-regulated kinases in cultured retinal pigment epithelial cells. Life Sci 2006; 79:545-50.

41. Yu JG, Javorschi S, Hevener AL, Kruszynska YT, Norman RA, Sinha M, et al. The effect of thiazolidinediones on plasma adiponectin levels in normal, obese and type 2 diabetic subjects. Diabetes 2002; 51:296874.

42. Higuchi A, Ohashi K, Shibata R, Sono-Romanelli $\mathrm{S}$, Walsh K, Ouchi N. Thiazolidinediones reduce pathological neovascularization in ischemic retina via an adiponectin-dependent mechanism. Arterioscler Thromb Vasc Biol 2010; 30:46-53.

43. Ouchi N, Kihara S, Arita Y, Nishida M, Matsuyama A, Okamoto Y, et al. Adipocyte-derived plasma protein, adiponectin, suppresses lipid accumulation and class A scavenger receptor expression in human monocytederived macrophages. Circulation 2001; 103:1057-63.

44. Ouchi N, Kihara S, Arita Y, Maeda K, Kuriyama H, Okamoto Y, et al. Novel modulator for endothelial adhesion molecules: adipocyte-derived plasma protein adiponectin. Circulation 1999; 100:2473-6.

45. Fernandez-Real JM, Botas-Cervero P, Lopez-Bermano A, Casamitjana R, Funahashi T, Delgado E, et al. Adiponectin is independently associated with glycosylated haemoglobin. Eur J Endocrinol 2004; 150:201-5.

46. Ouedraogo R, Wu X, Xu SQ, Fuchsel L, Motoshima $\mathrm{H}$, Mahadev K, et al. Adiponectin suppression of highglucose-induced reactive oxygen species in vascular endothelial cells: evidence for involvement of a cAMP signaling pathway. Diabetes 2006; 55:1840-6.

47. Motoshima H, Wu X, Mahadev K, Goldstein BJ. Adiponectin suppresses proliferation and superoxide generation and enhances eNOS activity in endothelial cells treated with oxidized LDL. Biochem Biophys Res Commun 2004; 315:264-71
48. Ouchi N, Kihara S, Arita Y, Okamoto Y, Maeda K, Kuriyama $\mathrm{H}$, et al. Adiponectin, an adipocyte-derived plasma protein, inhibits endothelial NFkappaB signaling through a cAMP-dependent pathway. Circulation 2000; 102:1296-301.

49. Matsuzawa Y. Therapy Insight: adipocytokines in metabolic syndrome and related cardiovascular disease. Nat Clin Pract Cardiovasc Med 2006; 3:35-42.

50. Yamagishi S, Adachi H, Abe A, Yashiro T, Enomoto $\mathrm{M}$, Furuki K, et al. Elevated serum levels of pigment epithelium-derived factor in the metabolic syndrome. J Clin Endocrinol Metab 2006; 91:2447-50.

51. Yano K, Bauchat JR, Liimatta MB, Clemmons DR, Duan C. Downregulation of protein kinase $\mathrm{C}$ inhibits insulin-like growth factor I-induced vascular smooth muscle cell proliferation, migration and gene expression. Endocrinology 1999; 140:4622-32.

52. Kowluru RA, Odenbach S. Role of interleukin-1beta in the pathogenesis of diabetic retinopathy. $\mathrm{Br}$ Ophthalmol 2004; 88:1343-7.

53. Carmo A, Cunha-Vaz JG, Carvalho AP, Lopes MC. $\mathrm{L}$-arginine transport in retinas from streptozotocin diabetic rats; correlation with the level of IL-1 $\beta$ and NO synthase activity. Vision Research 1999; 39:3817-23.

54. Romeo G, Liu WH, Asnaghi V, Kern TS, Lorenzi M. Activation of nuclear factork $\mathrm{B}$ induced by diabetes and high glucose regulates a proapoptotic program in retinal pericytes. Diabetes 2002; 51:2241-8.

55. Dinarello CA, Donath MY, Mandrup-Poulsen T. Role of Il-1beta in type 2 diabetes. Curr Opin Endocrinol Diabetes Obes 2010; 17:314-21.

56. Zhou L, Sun H, Xu J, Kang J. Level of vascular endothelial growth factor and interleukin- 6 in aqueous humor in diabetic retinopathy patients. Yan Ke XueBao 2010; 25:26-30.

57. Lee JH, Lee W, Kwon OH, Kim JH, Kwon OW, Kim $\mathrm{KH}$, et al. Cytokine profile of peripheral blood in type 2 diabetes mellitus patients with diabetic retinopathy. Ann Clin Lab Sci 2006; 38:361-7.

58. Rojas M, Zhang W, Lee D, Romero M, Nguyen DT, Al-Shabrawey M, et al. Role of IL-6 in angiotensin II-induced retinal vascular inflammation. Invest Ophthalmol Vis Sci 2010; 51:1709-18.

59. Nakazawa T, Hisatomi T, Nakazawa C, Noda K Maruyama K, She H, et al. Monocyte chemoattractant protein 1 mediates retinal detachment-induced photoreceptor apoptosis. Proc Natl Acad Sci USA 2007; 104:2425-30.

60. Oh IK, Kim SW, Oh J, Lee TS, Huh K. Inflammatory and angiogenic factors in the aqueous humor and the relationship to diabetic retinopathy. Curr Eye Res 2010; 35:1116-27.

61. Siebenlist U, Franzoso G, Brown K. Structure, regulation and function of NFKB. Annu Rev Cell Biol 1994; 10:405-55.

62. Hayden MR, Yang Y, Habibi J, Sowers JR. Pericytopathy: oxidative stress and impaired cellular longevity in the pancreas and skeletal muscle in metabolic syndrome and type 2 diabetes. Oxid Med Cell Longev 2010; 3:290-303.

63. Maiese K. Ringing in the New Year: new prospects for development, aging and longevity. Oxid Med Cell Longev 2010; 3:1. 


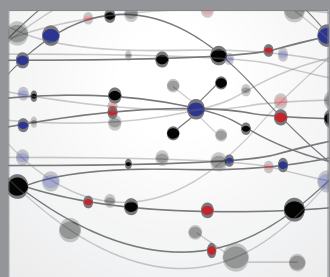

The Scientific World Journal
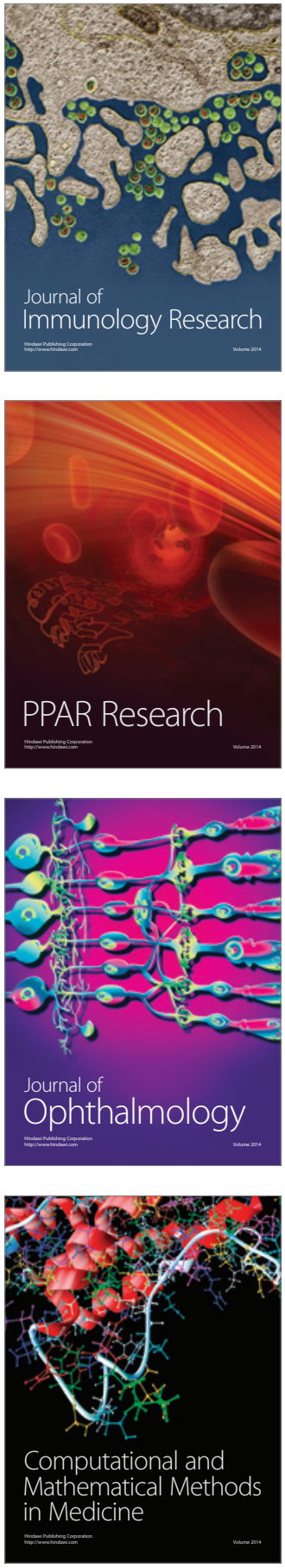

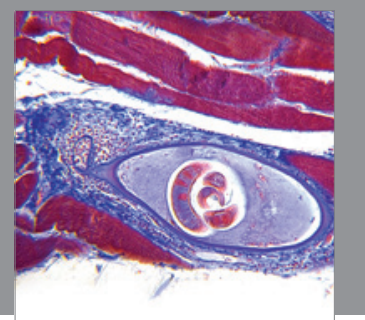

Gastroenterology

Research and Practice
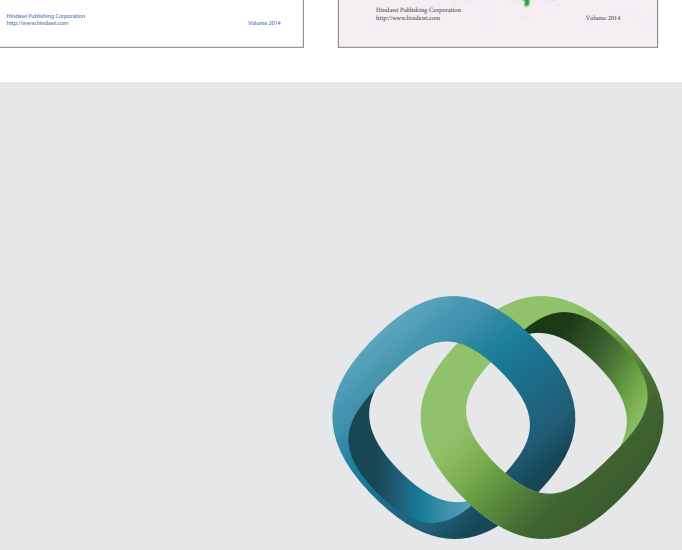

\section{Hindawi}

Submit your manuscripts at

http://www.hindawi.com
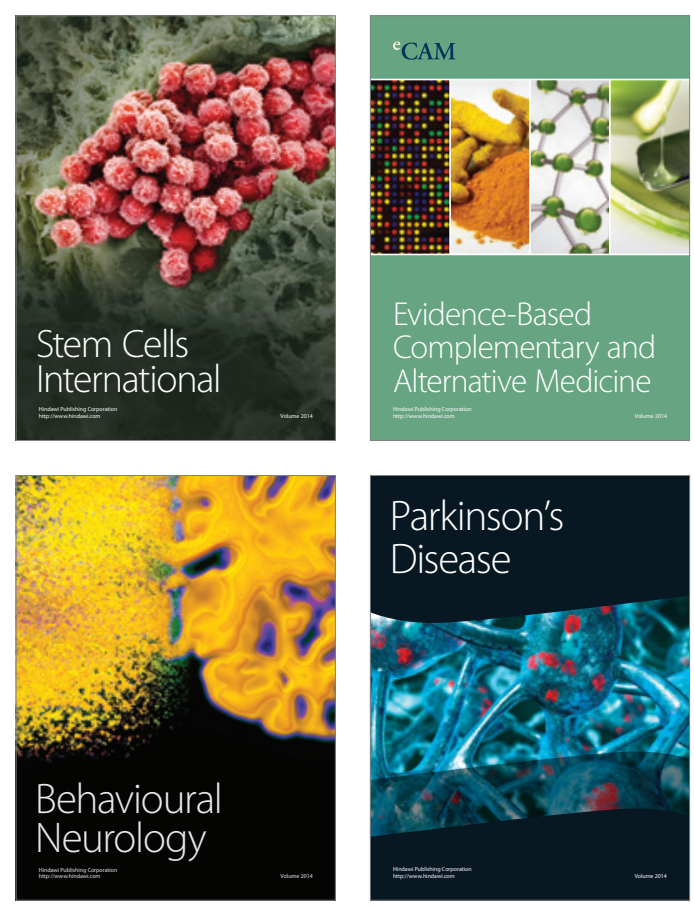

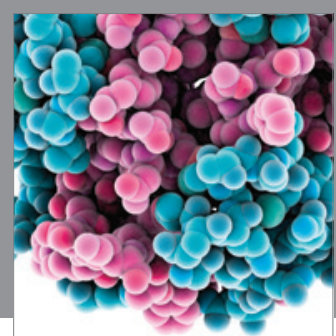

Journal of
Diabetes Research

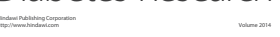

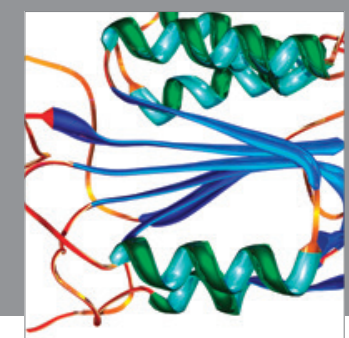

Disease Markers
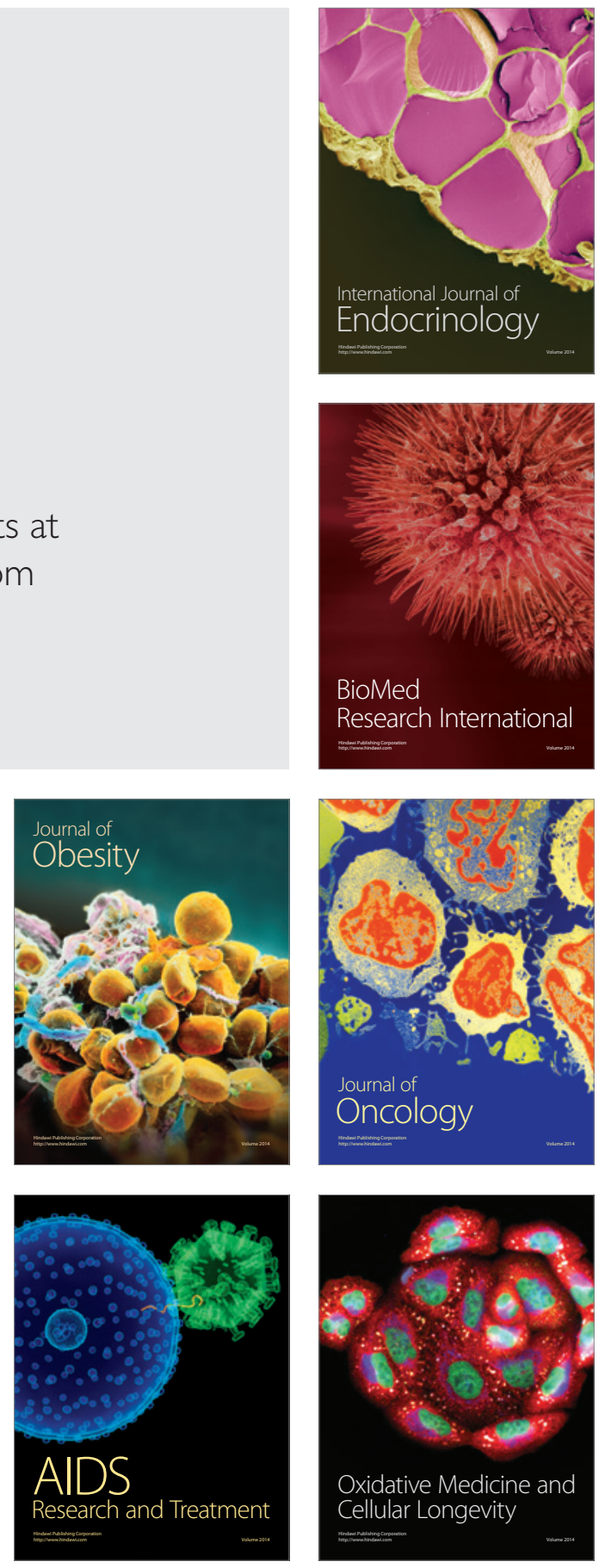